\title{
Chiral Lagrangian with confinement from the QCD Lagrangian
}

\author{
Yu.A.Simonov \\ Jefferson Laboratory,Newport News,VA 23606,USA , \\ State Research Center, \\ Institute of Theoretical and Experimental Physics, Moscow, Russia
}

November 1, 2018

\begin{abstract}
An effective Lagrangian for the light quark in the field of a static source is derived systematically using the exact field correlator expansion. The lowest Gaussian term is bosonized using nonlocal colorless bosonic fields and a general structure of effective chiral Lagrangian is obtained containing all set of fields. The new and crucial result is that the condensation of scalar isoscalar field which is a usual onset of chiral symmetry breaking and is constant in space-time, assumes here the form of the confining string and contributes to the confining potential, while the rest bosonic fields describe mesons with the $q \bar{q}$ quark structure and pseudoscalars play the role of Nambu-Goldstone fields. Using derivative expansion the effective chiral Lagrangian is deduced containing both confinement and chiral effects for heavy-light mesons. The pseudovector quark coupling constant is computed to be exactly unity in the local limit,in agreement with earlier large $N_{c}$ arguments.
\end{abstract}

\section{Introduction}

It was understood long ago [1] that chiral symmetry breaking in QCD is responsible for the low mass of pions and therefore the low-energy limit of QCD can be adequately described by the effective chiral Lagrangians [2].

In this approach the Nambu-Goldstone particles are described by local field variables and the resulting Effective Chiral Lagrangian (ECL) is local.

The most general and practically useful form of chiral Lagrangian was given in [3] and contains around ten phenomenological parameters ( 14 to the 4 th order in $p$ ), to be found from experiment.

Being successful in describing low-energy processes with Nabmu-Goldstone mesons, ECL has two major defects: Firstly, it does not take into account the quark structure of mesons, and consequently e.g. the formfactor computed in ECL can display in the meson only mesonic degrees of freedom. 
Secondly, ECL completely disregards the phenomenon of confinement, which is also important at small energies, and hence degrees of freedom of vacuum gluons, creating confining string are not taken into account.

There have been attempts to cure the first defect, namely the model Lagrangians have been suggested which take into account both quarks and mesons [4, 5]. In particular the instanton model of the QCD vacuum has been used to derive the ECL and the QuarkMeson Lagrangian(QML) [6]. As a result interesting interconnections of quark and chiral degrees of freedom have been demonstrated in the example of the nucleon [7].

However instantons are suppressed in the realistic vacuum of QCD [8, 9] and moreover the internal consistency of the instanton vacuum without confinement is seriously questioned [10, 11.

Moreover instantonic vacuum lacks confinement and therefore cannot cure the second defect of ECL and QML, therefore model ECL and QML obtained in [6, 7] disregard confinement fully.

Finally, the Effective Quark Lagrangian (EQL) obtained after averaging over gluon degrees of freedom, contains in principle infinite number of terms with growing number of quark fields. It was shown in [12] that specifically for instanton vacuum all higher terms are of the same order, while only the lowest term (so-called 'tHooft Lagrangian) is taken in the standard instanton lore.

It is the purpose of the present paper to start a new and systematic approach to the derivation of QML and ECL from the first principles - the QCD Lagrangian. In doing so the most important is to keep gauge invariance at every stage, therefore we shall consider the simplest gauge-invariant system of a light guark in the field of a static antiquark (generalization to only light quark systems will be done later).

After averaging over gluon fields one obtains EQL with infinite number of terms, containing as kernels irreducible gluon Field Correlators (FC). Recent measurements for the realistic QCD vacuum have shown that FC create a hierarchy, where the lowest (Gaussian) correlator is dominating [8, 9], while the next (4th order) correlator contributes around $1 \%$ to the static $Q \bar{Q}$ interaction (note that this situation is drastically different from that of instantonic vacuum, where higher correlators are equally important). We assume that a similar hierarchy should be present also in our problem of a light-heavy quark, which allows us to study the resulting EQL term by term, paying most attention to the lowest, $4 q$-piece. The next step is the bosonization procedure, i.e. an identical transformation introducing nonlocal colorless bosonic fields, having different Lorentz and flavour indices due to the use of Fierz transformation.

A special attention deserves the $6 q$ term, where bosonization may be done introducing bosonic fields for $\bar{q} q$ combination or else introducing baryonic fields for $3 q$ combination.

As a result one obtains QML or Quark-Baryon Lagrangian (QBL) in the most general and rigorous form. These questions will be considered in a separate publication [13].

As the next step one can use the stationary point analysis to obtain nonlinear equations for effective bosonic fields.

This is done in a rigorous way and the resulting equations contain for the scalarisoscalar part the same equation as was derived previously [14] in a gauge-invariant DysonSchwinger approach not using bosonization. The careful study of that equation in [14]16] has shown that it describes in the light-heavy system both confinement and chriral symmetry breaking, which coexist. This fact means that confinement in the language of 
effective meson fields, enters in the form of condensate of the scalar field inside the string, while other fields describe subdominant features of the $q \bar{Q}$ dynamics.

The paper is organized as follows. In the next section the averaging over vacuum gluonic fields is done and EQL is obtained. Special attention is devoted to the gauge invariance and parallel transporters necessary to ensure it for nonlocal $q \bar{q}$ combinations. In section 3 the bosonization procedure is done and Fierz transformation is introduced to obtain final QML with proper classification in Lorentz and flavour indices.

In section 4 the resulting ECL is obtained and the stationary point equations are derived and compared to the previously obtained in the Dyson-Schwinger approach. In section 5 the derivative expansion of the nonlocal bosonized Lagrangian is done and nonlocal forms of the lowest 2nd and 4th order terms in this expansion are obtained. Keeping only pion field in addition to quarks, one derives in section 6 the effective Lagrangian which appears to have the expected form with the pseudovector quark-pion coupling constant $g_{A}^{q}$. The latter is equal exactly to one, in agreement with earlier large $N_{c}$ argument, when local approximation is made. The concluding section is devoted to the discussion of confinement and chiral properties of the resulting ECL in the heavy-light meson case.

\section{The Effective Quark Lagrangian}

Consider the QCD partition function in the Euclidean space-time

$$
Z=\int D A D \psi D \psi^{+} e^{-S_{0}(A)+\int{ }^{f} \psi^{+}(i \hat{\partial}+i m+g \hat{A}){ }^{f} \psi d^{4} x}
$$

where $S_{0}(A)=\frac{1}{4} \int\left(F_{\mu \nu}^{a}(x)\right)^{2} d^{4} x, m$ is the current quark mass, and the quark operator ${ }^{f} \psi_{a \alpha}(x)$ has flavor index $f\left(f=1, \ldots n_{f}\right)$, color index $a\left(a=1, \ldots N_{c}\right)$ and Lorenz bispinor index $\alpha(\alpha=1,2,3,4)$.

The next step is to integrate over $D A_{\mu}$ with the weight $S_{0}(A)$, this is the gluon vacuum averaging which is denoted by \langle\rangle$_{A}$. Before doing this however one should choose the gauge-invariant system and using an appropriate gauge, express $A_{\mu}$ through the fieldstrength operator $F_{\mu \nu}$ which would finally appear in gauge-invariant combinations - Field Correlators (FC)(see [17] for review and more discussion), namely

$$
g^{n}\left\langle F_{\mu_{1} \nu_{1}}\left(x_{1}\right) \Phi_{C_{1}}\left(x_{1}, x_{2}\right) F_{\mu_{2} \nu_{2}}\left(x_{2}\right) \Phi_{C_{2}}\left(x_{2}, x_{3}\right), \ldots F_{\mu_{n} \nu_{n}}\left(x_{n}\right) \Phi C_{n}\left(x_{n}, x_{1}\right)\right\rangle_{A} \equiv \Delta^{(n)}
$$

where parallel transporters are defined as

$$
\Phi_{C}(x, y)=P \exp \left(i g \int_{C(x, y)} A_{\mu} d z_{\mu}\right)
$$

and the open contour $C(x, y)$ connects points $x$ and $y$ and can be arbitrary otherwise.

To achieve this goal one can use the so-called contour gauge [18, 19] which is especially convenient in the case of light quark moving in the field of a static antiquark. One has for the contour $z_{\mu}(s, x)$ starting at point $x$ and ending at $Y=z(0, x)$

$$
A_{\mu}(x)=\int_{0}^{1} d s \frac{\partial z_{\nu}(s, x)}{\partial s} \frac{\partial z_{\rho}(s, x)}{\partial x_{\mu}} F_{\nu \rho}(z(s)) \equiv \int_{Y}^{x} d \Gamma_{\mu \nu \rho}(z) F_{\nu \rho}(z) .
$$


In particular case, when the contour $z_{\mu}(s, x)$ goes along the shortest (straight) way to the $x_{4^{-}}$axis and then along this axis to some point $Y$, which can be at $-\infty$, one has the so-called modified Fock-Schwinger gauge [20], which was extensivelly used in [14] to get EQL. Here one has

$$
A_{\mu}\left(\mathbf{x}, x_{4}\right)=\int_{0}^{x_{i}} \alpha_{\mu}(u) d u_{i} F_{i \mu}\left(u, x_{4}\right),
$$

and $\alpha_{4}(u) \equiv 1$ while for $\mu=1,2,3, \alpha_{\mu}$ is equal to

$$
\alpha(u)=\frac{u_{i}}{x_{i}}, i=1,2,3
$$

It is convenient to write all expressions in a gauge-invariant way, using the property [19, that $\Phi_{C}$ given by (3) is identically equal to unity when the contour $C(x, y)$ lies on $z_{\mu}(s, x)$ or $z_{\mu}(s, y)$. Therefore one can define gauge-covariant operators referred to the point $Y$,

$$
\begin{gathered}
\psi^{(Y)}(x)=\Phi_{C(x, Y)} \psi(x) \equiv \Phi(Y, x) \psi(x) \\
\psi^{+(Y)}(x)=\psi^{+}(x) \Phi(x, Y) \\
F_{\mu \nu}^{(Y)}(x)=\Phi(Y, x) F_{\mu \nu}(x) \Phi(x, Y) .
\end{gathered}
$$

Here the contour $C(x, Y)$ in $\phi(Y, x)$ goes along $z(s, X)$ from $Y$ to $x$, and in opposite direction in $\Phi(x, Y)$.

For the field correlators referred to the same point $Y$ one can write $(a b, c d$-fundamental color indices, Lorentz indices are suppressed for simplicity reasons)

$$
\begin{gathered}
g^{2}\left\langle\left(F^{(Y)}(x)\right)_{a b}\left(F^{(Y)}(y)\right)_{c d}\right\rangle=\frac{\delta_{a d} \delta_{b c}}{N_{c}^{2}} g^{2}\left\langle\operatorname{tr}\left(F^{(Y)}(x) F^{(Y)}(y)\right)\right\rangle \\
g^{3}\left\langle\left(F^{(Y)}(x)\right)_{a b}\left(F^{Y}(y)\right)_{c d}\left(F^{(Y)}(z)\right)_{e f}\right\rangle=\delta_{b c} \delta_{d e} \delta_{a f}\left[-\frac{g^{3} \operatorname{tr}\langle x z y\rangle}{N_{c}\left(N_{c}^{4}-1\right)}+\right. \\
\left.+\frac{N_{c}}{N_{c}^{4}-1} g^{3} \operatorname{tr}\langle x y z\rangle\right]+\delta_{b e} \delta_{f c} \delta_{a d}\left[-\frac{g^{3} \operatorname{tr}\langle x y z\rangle}{N_{c}\left(N_{c}^{4}-1\right)}+\frac{N_{c} g^{3} t r\langle x z y\rangle}{N_{c}^{4}-1}\right]
\end{gathered}
$$

where notation is introduced e.g. $\operatorname{tr}\langle x y z\rangle \equiv\left\langle\operatorname{tr}\left(F^{(Y)}(x) F^{(Y)}(y) F^{(Y)}(z)\right)\right\rangle$.

Derivation of $(7),(8)$ is given in Appendix 1.

Let us first concentrate on the bilocal correlator (17). From (17), (5) it is clear that in the average value of $\left\langle A_{\mu}(x) A_{\nu}(y)\right\rangle$ the arguments of $F(z(s, x))$ and $F(z(s, y))$ are separated by the distance $r \sim T_{g}$, where $T_{g}$ is the gluonic correlation length [21], which was measured on the lattice [22] and estimated analytically [23] to be $T_{g} \sim 0.2 \mathrm{fm}$ (or even smaller, if data on gluelump masses [24 are used). For such distances $r \sim T_{g}$, which satisfy $r \ll|x-Y|,|y-Y|$ or for the gauge (5), $r \ll|\mathbf{x}|,|\mathbf{y}|$, one has an estimate

$$
\left\langle\operatorname{tr} F^{(Y)}(x) F^{(Y)}(y)\right\rangle=\langle\operatorname{tr}(F(x) \Phi(x, y) F(y) \Phi(y, x))\rangle+O\left(\frac{r^{2}}{\mathbf{x}^{2}}, \frac{r^{2}}{\mathbf{y}^{2}}\right)
$$

where the correlator on the r.h.s. of (9) is connected by straight lines from $x$ to $y$. A similar estimate holds for the triple FC (8), and in what follows we shall use the straightline form (9) which is independent on the position of the reference point $Y$. 
For that correlator one can use the general representation found in 25]

$$
\frac{g^{2}}{N_{c}}\left\langle\operatorname{tr} F_{\mu \nu}(x) \Phi(x, y) F_{\rho \sigma}(y) \Phi(y, x)\right\rangle=D(x-y)\left(\delta_{\mu \rho} \delta_{\nu \sigma}-\delta_{\mu \sigma} \delta_{\nu \rho}\right)+\Delta_{\mu \nu, \rho \sigma}^{(1)}
$$

where $\Delta^{(1)}$ has the structure of a full derivative and therefore does not contribute to confinement, its nonperturbative (NP) part is much smaller than that of $D$ and we shall omit it.

The function $D(u)$ has NP part which was measured in 22] and has exponential form,

$$
D(u)=D(0) \exp \left(-\frac{|u|}{T_{g}}\right)
$$

Finally, the string tension $\sigma$ can be expressed through $D(u)$ (and higher correlators) and at least for static quarks $D(u)$ yields dominant (up to few percent [8, 9]) contribution to $\sigma$,

$$
\sigma=\frac{1}{2} \int D(u) d^{2} u
$$

Having in mind all the above relations, one can now average over gluonic fields in (而) to obtain

$$
Z=\int D \psi D \psi^{+} e^{\int{ }^{f} \psi^{+}(i \hat{\partial}+i m)^{f}} \psi d^{4} x e^{L_{E Q L}^{(2)}+L_{E Q L}^{(3)}+\ldots}
$$

where the EQL proportional to $\left\langle\left\langle A^{n}\right\rangle\right\rangle$ is denoted by $L_{E Q L}^{(n)}$,

$$
\begin{gathered}
L_{E Q L}^{(2)}=\frac{g^{2}}{2} \int d^{4} x d^{4} y{ }^{f} \psi_{a \alpha}^{+}(x){ }^{f} \psi_{b \beta}(x){ }^{g} \psi_{c \gamma}^{+}(y){ }^{g} \psi_{d \varepsilon}(y)\left\langle A_{a b}^{(\mu)}(x) A_{c d}^{(\nu)}(y)\right\rangle \gamma_{\alpha \beta}^{(\mu)} \gamma_{\gamma \varepsilon}^{(\nu)} \\
L_{E Q L}^{(3)}=\frac{g^{3}}{3 !} \int d^{4} x d^{4} y d^{4} z{ }^{f} \psi_{a \alpha}^{+}(x){ }^{f} \psi_{b \beta}(x){ }^{g} \psi_{c \gamma}^{+}(y){ }^{g} \psi_{d \varepsilon}(y){ }^{h} \psi_{e \rho}^{+}(z){ }^{h} \psi_{f \sigma}(z) \times \\
\times\left\langle A_{a b}^{(\mu)}(x) A_{c d}^{(\nu)}(y) A_{e f}^{(\lambda)}(z)\right\rangle \gamma_{\alpha \beta}^{(\mu)} \gamma_{\gamma \varepsilon}^{(\nu)} \gamma_{\rho \sigma}^{(\lambda)}
\end{gathered}
$$

Average of gluonic fields can be computed using (5),(7), (8) as

$$
g^{2}\left\langle A_{a b}^{(\mu)}(x) A_{c d}^{(\nu)}(y)\right\rangle=\frac{\delta_{b c} \delta_{a d}}{N_{c}} \int_{0}^{x} d u_{i} \alpha_{\mu}(u) \int_{0}^{y} d v_{k} \alpha_{\nu}(v) D(u-v)\left(\delta_{\mu \nu} \delta_{i k}-\delta_{i \nu} \delta_{k \mu}\right) .
$$

The corresponding expression for the triple average $\left\langle A^{3}\right\rangle$ is given in Appendix 1 .

As it was argued in [26] the dominant contribution at large distances from the static antiquark is given by the color-electric fields, therefore we shall write down explicitly $L_{E Q L}^{(2)}(e l)$ for this case, i.e. taking $\mu=\nu=4$ in (14), (16), while the general case, also for generalized gauge (đ) is given in Appendix 2. As a result one has

$$
L_{E Q L}^{(2)}(e l)=\frac{1}{2 N_{c}} \int d^{4} x \int d^{4} y{ }^{f} \psi_{a \alpha}^{+}(x){ }^{f} \psi_{b \beta}(x){ }^{g} \psi_{b \gamma}^{+}(y){ }^{g} \psi_{a \varepsilon}(y) \gamma_{\alpha \beta}^{(4)} \gamma_{\gamma \varepsilon}^{(4)} J(x, y)
$$

where $J(x, y)$ is

$$
J(x, y)=\int_{0}^{x} d u_{i} \int_{0}^{y} d v_{i} D(u-v), \quad i=1,2,3 .
$$

Note that we have omitted everywhere for simplicity the upper index $(Y)$ in $\psi^{(Y)}(x)$, but it is implied, since otherwise both (14) and (15) are not gauge-invariant. 


\section{Bosonization of Effective Quark Lagrangians}

We shall separate out white bilinears in (17) following the standard procedure given for the general $L_{E Q L}^{(n)}$ in [14], [27],

$$
\Psi_{\alpha \varepsilon}^{f g}(x, y) \equiv{ }^{f} \psi_{a \alpha}^{+}(x){ }^{g} \psi_{a \varepsilon}(y)={ }^{f} \psi_{a^{\prime} \alpha}^{+}(x) \Phi_{a^{\prime} a}(x, Y) \Phi_{a c}(Y, y){ }^{g} \psi_{c \varepsilon}(y)
$$

and introduce the isospin generators $t_{f g}^{(n)}$

$$
\sum_{n=0}^{n_{f}^{2}-1} t_{f g}^{(n)} t_{i j}^{(n)}=\frac{1}{2} \delta_{f j} \delta_{g i} ; t^{(0)}=\frac{1}{\sqrt{2 n_{f}}} \hat{1} .
$$

Hence the bilinears in (17) can be written as

$$
\Psi_{\alpha \varepsilon}^{f g}(x, y) \Psi_{\gamma \beta}^{g f}(y, x)=2 \sum_{n=0}^{n_{f}^{2}-1} \Psi_{\alpha \varepsilon}^{(n)}(x, y) \Psi_{\gamma \beta}^{(n)}(y, x)
$$

where we have defined

$$
\Psi_{\alpha \varepsilon}^{(n)}(x, y) \equiv{ }^{f} \psi_{a \alpha}^{+}(x) t_{f g}^{(n)}{ }^{g} \psi_{a \varepsilon}(y) .
$$

Now one can use the Fierz transformation (see Appendix 3 for more details).

$$
\gamma_{\alpha \beta}^{(4)} \gamma_{\alpha^{\prime} \beta^{\prime}}^{(4)}=\frac{1}{4} \sum_{k=1}^{5} \bar{O}_{\alpha \beta^{\prime}}^{(k)} \bar{O}_{\alpha^{\prime} \beta}^{(k)}
$$

with

$$
\begin{gathered}
\bar{O}_{\alpha \beta}^{(1)}=\delta_{\alpha \beta}, \bar{O}^{(2)} \bar{O}^{(2)} \gamma^{(4)} \gamma^{(4)}-\gamma^{(i)} \gamma^{(i)}, \bar{O}^{(3)} \bar{O}^{(3)}=\left(\gamma^{5} \gamma^{(4)}\right)\left(\gamma^{5} \gamma^{(4)}\right)-\left(\gamma^{5} \gamma^{(i)}\right)\left(\gamma^{5} \gamma^{(i)}\right), \\
\bar{O}^{(4)} \bar{O}^{(0)}=\left(\sigma_{i k}\right)\left(\sigma_{i k}\right)-\left(\sigma_{4 k}\right)\left(\sigma_{4 k}\right)-\left(\sigma_{k 4}\right)\left(\sigma_{k 4}\right) \\
\bar{O}_{\alpha \beta}^{(5)}=i\left(\gamma^{(5)}\right)_{\alpha \beta} ; \quad \sigma_{\mu \nu}=\frac{\gamma_{\mu} \gamma_{\nu}-\gamma_{\nu} \gamma_{\mu}}{2 i} .
\end{gathered}
$$

Introducing (21) and (23) into (17), one obtains

$$
L_{E Q L}^{(2)}(e l)=-\int d^{4} x \int d^{4} y \Psi^{(n, k)}(x, y) \Psi^{(n . k)}(y, x) \tilde{J}(x, y)
$$

where we have defined

$$
\Psi^{(n, k)}(x, y)=\frac{1}{2} \Psi_{\alpha \varepsilon}^{(n)}(x, y) \bar{O}_{\varepsilon \alpha}^{(k)}, \quad \tilde{J} \equiv \frac{1}{N_{c}} J
$$

Bosonization is now done in a standard way using identity (signs and indices of summation and integration are suppressed)

$$
e^{-\Psi \tilde{J} \Psi}=\int(\operatorname{det} \tilde{J})^{1 / 2} D \chi \exp [-\chi \tilde{J} \chi+i \Psi \tilde{J} \chi+i \chi \tilde{J} \Psi]
$$

and hence the partition function with the only Gaussian contribution $L^{(2)}$ assumes the form

$$
Z=\int D \psi D \psi^{+} D \chi \exp L_{Q M L}
$$


where the effective quark-meson Lagrangian is

$$
\begin{gathered}
L_{Q M L}^{(2)}=\int d^{4} x \int d^{4} y\left\{{ }^{f} \psi_{a \alpha}^{+}(x)\left[(i \hat{\partial}+i m)_{\alpha \beta} \delta(x-y)+i M_{\alpha \beta}^{(f g)}(x, y)\right]{ }^{g} \psi_{a \beta}(y)-\right. \\
\left.-\chi^{(n, k)}(x, y) \tilde{J}(x, y) \chi^{(n, k)}(y, x)\right\}
\end{gathered}
$$

and the effective quark-mass operator is

$$
M_{\alpha \beta}^{(f g)}(x, y)=\sum_{n, k} \chi^{(n, k)}(x, y) \bar{O}_{\alpha \beta}^{(k)} t_{f g}^{(n)} \tilde{J}(x, y)
$$

In a similar way one can bosonize the term $L_{E Q L}^{(3)}$. However the computations are more lengthy and we shall present the result in a separate publication [13].

\section{The effective chiral Lagrangian $L_{E C L}$ and stationary point analysis}

We are now in position to integrate over quark fields in (28) and obtain Effective Chiral Lagrangian $L_{E C L}^{(2)}$. The result is

$$
Z=\int D \chi e^{L_{E C L}^{(2)}(\chi)}
$$

with

$$
\begin{aligned}
L_{E C L}^{(2)}(\chi) & =-\int d^{4} x d^{4} y \sum_{n, k} \chi^{(n, k)}(x, y) \tilde{J}(x, y) \chi^{(n, k)}(y, x)+ \\
& +N_{c} t r \ln [(i \hat{\partial}+i m) \delta(x-y)+i M(x, y)] .
\end{aligned}
$$

In (32) we have taken into account that $M$ is colorless, and the sign $t r$ refers to the summation (integration) over Lorentz, flavor indices and space-time coordinates.

As the next standard step one finds the stationary point equations to determine the ground state values for the auxiliary bosonic fields $\chi$. Taking functional derivative of $L_{E C L}^{(2)}$ with respect to $\chi^{(n, k)}$, one obtains

$$
\left.2 \chi^{(n, k)}(x, y) \tilde{J}(x, y)=-i N_{c} \operatorname{tr}[S(x, y)) \bar{O}^{(k)} t^{(n)}\right] \tilde{J}(x, y)
$$

where we have defined as in 14

$$
S(x, y)=-[(i \hat{\partial}+i m) \hat{1}+i \hat{M}]_{x, y}^{-1} .
$$

The set of (nonlinear) equations (33), (34) is the central result of the present paper. In what follows we shall study the properties of the solutions and compare this result to the previously obtained equations in [14], where another method was used -large $N_{c}$ approximation in the Dyson-Schwinger equations for the heavy-light system with one fixed flavour. In this case one should take $n_{f}=1$, and $t^{(0)}=\frac{1}{\sqrt{2}}$. Moreover, only scalar part (since it is dominant at large distances from the heavy source [14, 26] was considered, hence one can write instead of (30) 


$$
M \rightarrow M_{0}(x, y)=\frac{1}{\sqrt{2}} \chi(x, y) \tilde{J}(x, y)
$$

and (33) reduces to the equation

$$
i M_{0}(x, y)=\left(\gamma_{4} S(x, y) \gamma_{4}\right)_{k=1} J(x, y)
$$

where we have used the relation.

$$
\left(\gamma_{4} S \gamma_{4}\right)_{\alpha \gamma}=\gamma_{\alpha \beta^{\prime}}^{(4)} S_{\beta^{\prime} \alpha^{\prime}} \gamma_{\alpha^{\prime} \gamma}^{(4)}=\frac{1}{4} \sum_{k} O_{\alpha \gamma}^{(k)} \operatorname{tr}\left(S O^{(k)}\right) .
$$

The equation (36) is exactly the same as Eq. (15) in 14 where color-electric field component is retained, namely in the full answer

$$
i M_{0}(x, y)=J(x, y) \gamma_{\mu} S(x, y) \gamma_{\mu}-J_{i k} \gamma_{k} S(x, y) \gamma_{i}
$$

one keeps only $\mu=4, k=1$. As it is follows from the definition of $S$, Eq.(34), one has another equation to complete a full set

$$
(-i \hat{\partial}-i m) S(x, y)-i \int M(x, z) S(z, y) d^{4} z=\delta^{(4)}(x-y)
$$

Let us look more closely at the scalar part of mass operator, $M_{0}(x, y)$ Eqs. (35), (36). The properties of the kernel $J(x, y)$ (18) have been thoroughly investigated in [14- [16] and it was shown there, that when $\mathbf{x}$ is close to $\mathbf{y}$, then $J(x, y)$ is growing linearly at large $|\mathbf{x}|$, e.g. when for simplicity $D(u)$ is taken in the Gaussian form

$$
D(u)=D(0) e^{-\frac{\mathbf{u}^{2}+\mathbf{u}_{4}^{2}}{4 T_{g}^{2}}}
$$

then

$$
J(\mathbf{x} \sim \mathbf{y},|\mathbf{x}| \rightarrow \infty)=|\mathbf{x}| 2 T_{g} \sqrt{\pi} D(0) e^{-\left(x_{4}-y_{4}\right)^{2} / 4 T_{g}^{2}}
$$

whereas

$$
\sigma=\frac{1}{2} \int D(u) d^{2} u=2 \pi T_{g}^{2} D(0) .
$$

Moreover $S(x, y)$ (34) (where only $M_{0}$ is retained) at large distances displays the properties of the smeared $\delta$-function (see [14]-16] for discussion and numerical estimates)

$$
\gamma_{4} S(x, y) \gamma_{4} \sim \tilde{\delta}^{(3)}(\mathbf{x}-\mathbf{y})
$$

and as a result the product $J(x, y) \gamma_{4} S(x, y) \gamma_{4}$ behaves linearly in $|\mathbf{x}|$ at large $|\mathbf{x}|$, in such a way that in the equation (38) one has

$$
\int M_{0}(x, z) S(z, y) d^{4} z \rightarrow \sigma|\mathbf{x}| S(x, y)
$$

Thus for $|\mathbf{x}| \gg T_{g}$ one has

$$
M_{0}(x, z) \approx \sigma|\mathbf{x}| \tilde{\delta}^{(4)}(x-z)\left(1+O\left(T_{g} /|\mathbf{x}|\right)\right.
$$


and $\tilde{\delta}^{(4)}(x-z)$ is smeared off at distance of the order of $T_{g}$. To proceed we disregard first in the sum (30) all terms except for the scalar and pseudoscalar fields,

$$
M(x, y)=\chi_{S}(x, y) \frac{\tilde{J}(x, y)}{\sqrt{2 n_{f}}}+\hat{\chi}_{\pi}(x, y) i \gamma_{5} \tilde{J}(x, y)
$$

with

$$
\hat{\chi}_{\pi}(x, y)=\chi_{\pi}^{(f)} t^{f}
$$

The form (45) can be equivalently parametrized in a nonlinear way as follows

$$
\hat{M}(x, y)=M_{S}(x, y) \hat{U}(x, y), \hat{U}=\exp \left(i \gamma_{5} \hat{\phi}\right), \hat{\phi}(x, y)=\phi^{f}(x, y) t^{f} .
$$

Now using normalization property

$$
\operatorname{tr}\left(t^{(n)} t^{(m)}\right)=\frac{1}{2} \delta_{n m}, n, m=0,1, \ldots, n_{f}-1,
$$

one easily obtains

$$
\frac{1}{4} \operatorname{tr}\left(\hat{M} \hat{M}^{+}\right)=\frac{1}{2}\left(\chi_{s}^{2}+\vec{\chi}_{\pi}^{2}\right) \tilde{J}^{2}(x, y)=M_{S}^{2} n_{f}
$$

and hence the first term in (32) can be written as

$$
\left(\chi_{s}^{2}+\chi_{\pi}^{2}\right) \tilde{J}(x, y)=2 n_{f} \tilde{J}^{-1}(x, y) M_{S}^{2}(x, y)
$$

and the total Lagrangian (32) is

$$
L_{E C L}^{(2)}\left(M_{S}, \hat{\phi}\right)=-2 n_{f}(\tilde{J}(x, y))^{-1} M_{S}^{2}(x, y)+N_{c} \operatorname{tr} \log \left[(i \hat{\partial}+i m) \hat{1}+i M_{S} \hat{U}\right] .
$$

The stationary point equations assume the form

$$
\frac{\delta L_{E C L}^{(2)}}{\delta M_{S}(x, y)}=-4 n_{f}(\tilde{J}(x, y))^{-1} M_{S}(x, y)-N_{c} \operatorname{tr}\left(S i e^{i \gamma_{5} \hat{\phi}}\right)=0
$$

with

$$
\begin{gathered}
S(x, y)=-\left[i \hat{\partial}+i m+i M_{S} \hat{U}\right]_{x, y}^{-1} \\
\frac{\delta L_{E C L}^{(2)}}{\delta \hat{\phi}(x, y)}=N_{c} \operatorname{tr}\left(S M_{S} e^{i \gamma_{5} \hat{\phi}} \gamma_{5}\right) .
\end{gathered}
$$

The solution with $\hat{\phi}=0, M_{S}=M_{S}^{(0)} \operatorname{satisfies(53)}$ while(51) may be rewritten in the form

$$
i M_{S}^{(0)}(x, y)=\frac{N_{c}}{4} \operatorname{tr} S \tilde{J}(x, y)=N_{c}\left(\gamma_{4} S \gamma_{4}\right)_{s c} \tilde{J}(x, y)
$$

where $(\Gamma)_{s c}$ means the scalar part of operator,as defined in (37), and one can see that one recovers Eq.(36), derived before in [14] in a different formalism. 


\section{The derivative expansion of $L_{E C L}^{(2)}$}

In this chapter chiral Lagrangian will be written as a series in powers of derivatives of the field $\hat{U}(x, y)$. A similar procedure for the local case and in absence of confinement was systematically done in [5], [29]-[30], and more recently in [31]. For the case of instanton model it was done in [7]. In all cases at some moment the local limit of the resulting chiral Lagrangian is done, and confining properties of the kernel $M_{S}(x, y)$ are not taken into account. In what follows we keep both $\hat{\phi}$ and $M_{S}$ nonlocal and the confining property (44) is exploited. It should be noted that nonlocality of the field $\hat{\phi}$ is a necessary consequence of its quark-antiquark structure and this structure is lost when localization approximation is done. Since the radius of the pion is around $0.6 \mathrm{fm}$, it is only for small momenta that localization procedure is justified. We now turn to the second term on the r.h.s. of (50) which contains pionic field and make an expansion of its real part in powers of derivatives of the field $U$. Note that imaginary part of effective chiral Lagrangian was studied in [5], [7], 30] and it starts with the terms of the 5-th power in pionic field; it will not be studied below. Defining the real part of the pionic effective action $R e L_{e f f}[\pi]$, one has

$$
R e L_{e f f}[\pi]=-\frac{N_{c}}{2} \log \operatorname{det} \frac{D^{+} D}{D_{0}^{+} D_{0}}
$$

where notations are used

$$
D=i \hat{\partial}+i m+i M_{S} \hat{U}, D_{0}=i \hat{\partial}+i m+i M_{S}
$$

Moreover

$$
\begin{gathered}
D^{+} D=-\partial^{2}-\partial_{\mu}\left(M_{S} \hat{U}^{+}\right) \gamma_{\mu}+\left(m+M_{S}\right)^{2}+m M_{S}\left(\hat{U}^{+}+\hat{U}\right) \\
D_{0}^{+} D_{0}=-\partial^{2}+\left(m+M_{S}\right)^{2} .
\end{gathered}
$$

To simplify we put $m=0$ and rewrite (55) as

$$
R e L_{e f f}[\pi]=-\frac{N_{c}}{2} \operatorname{tr} \log \left(1-G_{0} \partial_{\mu} M_{S} \hat{U}^{+} \gamma_{\mu}\right),
$$

where we have defined

$$
G_{0}(x, y)=\left(-\partial^{2}+M_{S}^{2}\right)_{x, y}^{-1} .
$$

In (59) the sign $t r$ implies the sum over Lorentz and flavour indices and integral over coordinates, having in mind that every factor appearing in (59) under the logarithm sign should be considered as a matrix in the space-time coordinates. Expanding logarithm in (59) and keeping only 2nd and 4th order terms in $\partial_{\mu} \hat{U}^{+}$, one obtains

$$
\begin{gathered}
R e L_{e f f}[\pi]=N_{c} \overline{\operatorname{tr}} \operatorname{Re}\left[G_{0} \partial_{\mu} U^{+} G_{0} \partial_{\mu} U\right. \\
\left.+\frac{1}{2} G_{0} \partial_{\mu} U^{+} G_{0} \partial_{\nu} U G_{0} \partial_{\alpha} U^{+} G_{0} \partial_{\beta} U\left(\delta_{\mu \nu} \delta_{\alpha \beta}+\delta_{\mu \beta} \delta_{\nu \alpha}-\delta_{\mu \alpha} \delta_{\nu \beta}\right)\right]+\ldots
\end{gathered}
$$

Here $\overline{t r}$ implies summing over flavour indices and integration over coordinates, and $U=$ $M_{S}(x, y) e^{i \phi(x, y)}$. We consider now the first term on the r.h.s. of (61) and write explicitly the coordinate part of $\overline{t r}$

$$
R e L_{e f f}^{(2)}[\pi]=N_{c} t r_{f} \operatorname{Re}\left[\int d^{4} x G_{0}(x, y) \partial_{\mu} U^{+}(y, z) d^{4} z G_{0}(z, u) d^{4} u \partial_{\mu} U(u, x)\right] .
$$


In the limit, when $M_{S}(x, y)$ becomes local,

$$
U(x, y) \approx \tilde{\delta}^{4}(x-y) M_{S}(x) e^{I \hat{\phi}(x)}=\tilde{\delta}^{4}(x-y) U(x)
$$

one has

$$
R e L_{e f f}^{2}[\pi]=N_{c} t r_{f} \operatorname{Re}\left[\int d^{4} x d^{4} y G_{0}(x, y) \partial_{\mu} U^{+}(y) G_{0}(y, x) \partial_{\mu} U(x)\right] .
$$

One can see that Eq.(63) yields a nonlocal chiral Lagrangian, the nonlocality being given by the range of the quark Green's function $G_{0}(x, y)$. In case of a model without confinement, e.g.instanton model or NJL model, $G_{0}(x, y)$ would represent the free Green's function of a massive quark, with the constituent mass $\mu$ created by chiral symmetry breaking, $\mu \approx 0.3 \mathrm{GeV}$. Hence the range of nonlocality in this model case is large, and the derivative expansion in powers of $\partial U$, which is a standard systematic procedure in [5], [7], 30] is justified for small momenta $p \leq \mu$. At this point one should remember that in (61) - (63) both $M_{S}(x, y)$ and $U(x, y)$ (and hence $S(x, y), \mathrm{Eq}(34)$ ), are defined gaugeinvariantly with respect to the contour $Y$, which for simplicity was taken to be $x_{4}$ axis. Therefore the resulting string in $\hat{M}, M_{S}$ goes from the points $(x, y)$ (coinciding when $T_{g}$ tends to zero) to the contour $Y$. Physically it means, that the effective Lagrangian (62) describes the bosonic field (e.g.pion) in the presence of the heavy quark. It will enable us to define in the following chapter matrix elements of heavy-light meson transitions with emission of a pion. The case of effective boson Lagrangian for light quarks and antiquarks (i.e. without the heavy quark line) will be considered in the second paper of this series.

\section{Pionic transitions in heavy-light mesons}

In this section the main emphasis will be on the pionic part of the quark mass operator, which enters the quark-meson Lagrangian as

$$
\Delta L=i \int d^{4} x d^{4} y \psi^{+}(x) \hat{M}(x, y) \psi(y)
$$

where $\hat{M}(x, y)$ according to (46) can be written in the form

$$
\hat{M}(x, y)=M_{S}(x, y) e^{i \gamma_{5} \hat{\phi}(x, y)} .
$$

In the limit of small $T_{g}$ one obtains for $M_{S}(x, y)$ a localized expression

$$
M_{S}(x, y) \approx \sigma|\mathbf{x}| \delta^{(4)}(x-y),|\mathbf{x}| \gg T_{g}
$$

and the corresponding linearized in $\hat{\phi}$ Lagrangian is (in the Minkowskian space-time)

$$
\Delta L^{(1)}=\int \bar{\psi}(x) \sigma|\mathbf{x}| \gamma_{5} \frac{\pi^{A} \lambda^{A}}{F_{\pi}} \psi(x) d t d^{3} x
$$

where $F_{\pi}$ is known [3] to be $F_{\pi}=94 \mathrm{MeV}$. For the pionic transition between heavy-light states one should compute matrix elements

$$
\left\langle M_{2}\left(\mathbf{p}_{2}\right), \pi(\mathbf{k})\left|\Delta L^{(1)}\right| M_{1}\left(\mathbf{p}_{1}\right)\right\rangle .
$$


Neglecting recoil momentum of heavy-light meson one effectively reduces the problem to the calculation of the matrix element

$$
W_{21}=\int \bar{\psi}_{2}(x) \frac{\sigma|\mathbf{x}|}{F_{\pi}} \gamma_{5} \frac{\lambda^{A} e^{i \mathbf{k x}}}{\sqrt{2 \omega_{\pi}(\mathbf{k}) V}} \psi_{1}(\mathbf{x}) d^{3} x
$$

and the decay probability is

$$
w=2 \pi\left|W_{21}\right|^{2} \delta\left(E_{1}-E_{2}-\omega\right) \frac{V d^{3} k}{(2 \pi)^{3}} .
$$

In (66) $\bar{\psi}_{2}(x), \psi_{1}(x)$ are quark wave functions of the heavy-light states 2 and 1 respectively,which can be taken from the solutions of the corresponding Dirac equations, found in [32], [26]. At this point one can rewrite the matrix element of $\Delta L^{(1)}$ between two stationary quark states $\psi_{m}, \psi_{n}$. Using Dirac equations

$$
\begin{gathered}
{\left[\boldsymbol{\alpha} \mathbf{p}+\beta(m+\sigma|\mathbf{x}|)+V_{\text {Coul }}\right] \psi_{n}=\epsilon_{n} \psi_{n}} \\
\bar{\psi}_{m}\left[-\boldsymbol{\alpha} \mathbf{p}+\beta(m+\sigma|\mathbf{x}|)+V_{\text {Coul }}\right]=\epsilon_{m} \bar{\psi}_{m}
\end{gathered}
$$

one obtains

$$
\left\langle m\left|\Delta L^{(1)}\right| n\right\rangle=\frac{1}{2 F_{\pi}}\left\langle m\left|-2 m \gamma_{5} \hat{\pi}+\beta \gamma_{5}\left(\epsilon_{m}-\epsilon_{n}\right) \hat{\pi}+\gamma_{5} \beta \boldsymbol{\alpha} \mathbf{p} \hat{\pi}\right| n\right\rangle .
$$

In the chiral limit, $m=0$, one can rewrite the last two terms inside the brackets in (73) as

$$
\gamma_{5}\left(\gamma \frac{\partial \hat{\pi}}{\partial \mathbf{x}}+i \beta \frac{\partial \hat{\pi}}{\partial t}\right)=\gamma_{5} \gamma_{\mu} \partial_{\mu} \hat{\pi}
$$

One obtains from (73) the form of the quark-pion interaction which one usually writes as (see [33, [34] and refs. therein)

$$
\Delta L^{c h}=g_{A}^{q} \operatorname{tr}\left(\bar{\psi} \gamma_{\mu} \gamma_{5} \omega_{\mu} \psi\right), \omega=\frac{i}{2 F_{\pi}}\left(u \partial_{\mu} u^{+}-u^{+} \partial_{\mu} u\right)
$$

where $u=\sqrt{\hat{U}}$ and $g_{A}^{q}$ is the axial vector coupling of the (constituent) quark. It is easy to see that $(\sqrt{73})$ is the first term in the expansion of $(\overline{75})$ in powers of the field $\hat{\pi}$, and it is gratifying that the parameter $g_{A}^{q}$ is defined theoretically to be equal exactly to 1 in our local approximation, which is in agreement with large $N_{c}$ argument in [33].

\section{Concluding remarks}

We have performed a systematic bosonization procedure starting from the QCD Lagrangian and have derived the nonlocal chiral Lagrangian and its limiting local form for quarks interacting with pionic field. By the method of construction, the resulting Lagrangian is applicable for quark-pion interaction when the quark is coupled by the string to the heavy antiquark. Therefore our results can be immediately applied to the pionic transitions in the heavy-light mesons. The one-pion transitions have been studied using 
the form (75) in [34], and it was found from comparison to experiment that the values of $g_{A}^{q}$ around 0.7 are preferred. It makes it reasonable to study the nonlocal version of the chiral Lagrangian (73), since nonlocality effectively decreases resulting matrix elements, possibly explaining the mismatch between our theoretical value $g_{A}^{q}=1$ and observed value of 0.7 . In a similar way one can obtain matrix elements for double pion emission in heavy boson transitions, which are of special interest for $(B, D),(B, D *)$ semileptonic decays. The case of purely light mesons, can be treated in a similar way, but needs another string configurations to be taken into account. This is planned to do in the subsequent paper of this series. The author is grateful to F.Gross and I.Musatov for discussions and to J.Goity for useful criticism and suggestions. This work was supported by DOE contract DE-AC05-84ER40150 under which SURA operates the Thomas Jefferson National Accelerator Facility.

\section{Appendix 1}

\section{Vacuum averages of Field Correlators}

For the field operator transported to the point $Y$,

$$
F_{\mu \nu}^{(Y)}(x)=\Phi(Y, x) F_{\mu \nu}(x) \Phi(x, Y)
$$

so that gauge transformation has the form

$$
F_{\mu \nu}^{(Y)}(x) \rightarrow U^{+}(Y) F_{\mu \nu}^{(Y)}(x) U(Y)
$$

the vacuum average of any product can be expressed through Kronecker symbols, e.g.

$$
g^{2}\left\langle\left(F^{(Y)}(x)\right)_{a b}\left(F^{(Y)}(y)\right)_{c d}\right\rangle=\delta_{a d} \delta_{b c} \mathcal{P}(x, y)
$$

where $a b, c d$ are fundamental color indices and $\mathcal{P}(x, y)$ can be easily connected to the color trace, i.e. to the FC,

$$
\mathcal{P}(x, y)=\frac{\delta_{a d} \delta_{b c}}{N_{c}^{2}} g^{2}\left\langle\operatorname{tr}\left(F^{(Y)}(x) F^{(Y)}(y)\right)\right\rangle .
$$

Similar rules apply to products of any number of $F^{(Y)}$, they can be deduced from the global $S U\left(N_{c}\right)$ rules for the tensors averaged with the Haar measure, namely one can make a gauge transformation

$$
\left(F_{\mu \nu}^{Y}(x)\right)_{a b} \rightarrow \Omega^{+}(Y)_{a c}\left(F_{\mu \nu}^{Y}(x)\right)_{c d} \Omega(Y)_{d b}
$$

and average over $D \Omega$ with the usual Haar measure. In this way one obtains relation (8). At this point it is interesting to note that the product of three F's may have another representation in the $S U(3)$ group, since there one can use totally antisymmetric tensor $e_{a b c}$. 
E.g. one can write the following equality

$$
\begin{gathered}
e_{a c e} e_{b d f}=\delta_{a b}\left(\delta_{c d} \delta_{e f}-\delta_{c f} \delta_{e d}+\right. \\
+\delta_{a d}\left(\delta_{c f} \delta_{e b}-\delta_{c b} \delta_{e f}\right)+ \\
+\delta_{a f}\left(\delta_{c b} \delta_{e d}-\delta_{c d} \delta_{e b}\right)
\end{gathered}
$$

Therefore on can use two forms of writing for the product. In the first case one writes

$$
\begin{gathered}
\left\langle\left(F^{(Y)}(x)\right)_{a b}\left(F^{(Y)}(y)\right)_{c d}\left(F^{(Y)}(z)\right)_{e f}\right\rangle=\mathcal{P}_{1}(x, y, z) \delta_{b c} \delta_{d e} \delta_{a f}+ \\
\mathcal{P}_{2}(x, y, z) \delta_{b c} \delta_{f c} \delta_{a d}
\end{gathered}
$$

and finds $\mathcal{P}_{1}, \mathcal{P}_{2}$ by multiplying both sides of (A1.7) with the corresponding contribution of $\delta$-symbols. In this way one arreves at the Eq. (8) in the main text. If instead one uses (A1.6) instead of one of combinations, or separates (A1.6) out of Eq. (\$), one arrives at the white $(3 q),(3 \bar{q})$ combinations.

\section{Appendix 2}

\section{Vacuum averages of $\left\langle(A)^{n}\right\rangle$}

One can use the generalized contour gauge expression for $A_{\mu}(x)$.

$$
A_{\mu}(x)=\int_{Y}^{x} d z_{\nu} \frac{\partial z_{\rho}}{\partial x_{\mu}} F_{\nu \rho}(z) \equiv \int_{Y}^{x} d \Gamma_{\mu \nu \rho}(z) F_{\nu \rho}(z)
$$

to represent the average of the product of any number of operators $A_{\mu}$ as

$$
\begin{gathered}
\left\langle\left(A_{\mu_{1}}^{\left(x_{1}\right)}\right)_{a_{1} b_{1} \ldots} \ldots\left(A_{\mu_{n}}\left(x_{n}\right)\right)_{a_{n} b_{n}}\right\rangle= \\
=\int_{Y}^{x_{1}} d \Gamma_{\mu_{1} \nu_{1} \rho_{1}}\left(z_{1}\right) \ldots \int_{Y}^{x_{n}} d \Gamma_{\mu_{n} \nu_{n} \rho_{n}}\left(z_{n}\right)\left\langle\left(F_{\nu_{1} \rho_{1}}^{(Y)}\left(z_{1}\right)\right)_{a_{1} b_{1} \ldots} \ldots\left(F_{\nu_{n} \rho_{n}}^{(Y)}\left(z_{n}\right)\right)_{a_{n} b_{n}}\right\rangle .
\end{gathered}
$$

In a particular case of the modified Fock-Schwinger gauge one has: $Y=0, x_{i} \rightarrow \mathbf{x}_{i}$

$$
d \Gamma_{\mu \nu \rho}(z)=\alpha_{\mu}(z) d z_{\nu} \delta_{\rho \mu}
$$

\section{Appendix 3}

\section{Fierz transformations}

In this appendix the derivation is given of Fierz tranformations for combinations of $\gamma_{\mu}$ matrices met in the text above. It is based on the clear presentation done in the book [29. Note however that we are always working with the Euclidean $\gamma$ matrices, therefore some details and coefficients obtained below are different from [29]. 
We start with the general expansion for any $4 \times 4$ matrix

$$
\begin{aligned}
& \gamma=\frac{1}{4} \sum_{A} C_{A} \gamma_{A}, \quad A=1, \ldots 16, \\
& \gamma_{A}=1, \quad A=1 \\
& \gamma_{A}=\gamma_{\mu} \\
& \mu=1,2,3,4 ; \quad A=2,3,4,5 \\
& \gamma_{A}=\sigma_{\mu \nu}=\frac{\gamma_{\mu} \gamma_{\nu}-\gamma_{\nu} \gamma_{\mu}}{2 i}, \\
& A=6,7,8,9,10,11 \\
& \gamma_{A}=\gamma_{5} \gamma_{\mu} \text {, } \\
& A=12,13,14,15 \\
& \gamma_{A}=\gamma_{5} \\
& A=16
\end{aligned}
$$

In (A3.1) one can derive the general representation for any matrices $F_{m k}, G_{i l}$, indeed from relation

$$
\frac{1}{4} \sum_{A} \Delta_{A} \gamma_{m l}^{A} \gamma_{i k}^{A}=\delta_{m k} \delta i l
$$

changing $m \rightarrow m^{\prime}, l \rightarrow l^{\prime}$ and multiplying with $F_{m m^{\prime}} G_{l^{\prime} l}$ one obtains

$$
F_{m k} G_{i l}=\frac{1}{4} \sum_{A} \Delta_{A}\left(F \gamma_{A} G\right)_{m l}\left(\gamma_{A}\right)_{i k} .
$$

For Euclidean matrices $\gamma_{A}$ one easily obtains $\Delta_{a}=-1$, for $A=12,13,14,15$ and $\Delta_{A}=1$ otherwise. Taking $F=G=\gamma^{4}$, one obtains

$$
\begin{gathered}
\gamma_{m k}^{4} \gamma_{i l}^{4}=\frac{1}{4} \sum_{A} \Delta_{A}\left(\gamma^{4} \gamma_{A} \gamma^{4}\right)_{m l}\left(\gamma_{A}\right)_{i k}= \\
=\frac{1}{4}\left\{(1)(1)+\left(\gamma^{4}\right)\left(\gamma^{4}\right)-\left(\gamma^{i}\right)\left(\gamma^{i}\right)+\left(\sigma^{i k}\right)\left(\sigma^{i k}\right)-\left(\sigma^{4 k}\right)\left(\sigma^{4 k}\right)-\right. \\
\left.-\left(\sigma^{k 4}\right)\left(\sigma^{k 4}\right)+\left(\gamma^{5} \gamma^{4}\right)\left(\gamma^{5} \gamma^{4}\right)-\left(\gamma^{5} \gamma^{i}\right)\left(\gamma^{5} \gamma^{i}\right)-\left(\gamma^{5}\right)\left(\gamma^{5}\right)\right\}= \\
\frac{1}{4} \sum_{k=1}^{5} \bar{O}_{m l}^{(k)} \bar{O}_{i k}^{(k)} .
\end{gathered}
$$

The last relation coincides with (23) and operators $\bar{O}^{(k)}$ are given in (24). Note that in the curly brackets in (A3.4) each product of $\gamma$ matrices, $(\gamma \gamma)(\gamma \gamma)$, has the same order of indices as in $\bar{O}_{m l}^{(k)} \bar{O}_{i k}^{(k)}$. In a similar way one can represent the combination of spacial $\gamma$ matrices, $n=1,2,3$, no summation over $n$,

$$
\begin{gathered}
\gamma_{m k}^{n} \gamma_{i l}^{n}=\frac{1}{4}\left\{(1)(1)+\left(\gamma^{4}\right)\left(\gamma^{4}\right)-\left(\gamma^{m}\right)\left(\gamma^{m}\right)+\left(\gamma^{n}\right)\left(\gamma^{n}\right)+\right. \\
+\left(\sigma^{\mu \nu}\right)\left(\sigma^{\mu \nu}\right)_{n \neq \mu \nu}-\left(\sigma^{n \nu}\right)\left(\sigma^{n \nu}\right)-\left(\sigma^{\nu n}\right)\left(\sigma^{\nu n}\right)-\left(\gamma^{5} \gamma^{\mu}\right)\left(\gamma^{5} \gamma^{\mu}\right)_{\mu \neq n}+ \\
\left.+\left(\gamma^{5} \gamma^{n}\right)\left(\gamma^{5} \gamma^{n}\right)-\left(\gamma^{5}\right)\left(\gamma^{5}\right)\right\}_{m l, i k} .
\end{gathered}
$$

Summing (A3.5) over $n$ and adding (A3.4) one obtains

$$
\left(\gamma^{\mu}\right)_{m k}\left(\gamma^{\mu}\right)_{i l}=\left\{(1)(1)-\frac{1}{2}\left(\gamma^{\mu}\right)\left(\gamma^{\mu}\right)-\frac{1}{2}\left(\gamma^{5} \gamma^{\mu}\right)\left(\gamma^{5} \gamma^{\mu}\right)-\left(\gamma^{5}\right)\left(\gamma^{5}\right)\right\}_{m l, i k}
$$

Now in case of generalized contour gauge as in Appendix 2 one has to make Fierz transformation of the combination

$$
\left(\gamma^{\mu}\right)_{m k}\left(\gamma^{\nu}\right)_{i l}=\frac{1}{4} \sum_{A} \Delta_{A}\left(\gamma^{\mu} \gamma_{A} \gamma^{\nu}\right)_{m l}\left(\gamma_{A}\right)_{i k}
$$

Note that scalar and pseudoscalar combinations occur on the l.h.s. of (A3.7) only for $\mu=\nu$. 


\section{References}

[1] Y.Nambu, Phys. Rew. Lett. 4 (1960) 380; Chjou Guanjao ZhETF 39 (1960) 703.

[2] S.Weinberg, The Quantum Theory of Fields,vol.2,Univ.Press,1995.

[3] J.Gasser and H.Leutwyler, Nucl. Phys. B250 (1985) 465

[4] M.K.Volkov and D.Ebert, Sov. J. Nucl. Phys. 36 (1982) 736; Z.Phys. C16(1983) 205

[5] D.I.Diakonov, M.I.Eides, JETP Lett 38 (1983) 433;

M.S.Birse, M.K.Banarjee, Phys. Lett. B136 (1984) 284;

I.J.R.Aitchison, C.M.Frazer, Phys. Lett. B146 (1984) 63: Phys. Rev. D31 (1985) 2605: ibid. D32 (1985) 2190;

R.MacKenzie, F.Wilczek, A.Zee, Phys. Rev. Lett. 53 (1984) 2203;

S.Kahana, G.Ripka, Nucl. Phys. A415 (1984) 351;

Kahana, G.Ripka, Nucl. Phys. A429 (1984) 462;

A.Niemi, Phys. Rev. Lett. 54 (1985) 631;

Lai-Him Chan, Phys. Rev. Lett. 55 (1985) 21;

A.Dhar and S.Wadia, Phys. Rev. Lett. 52 (1984) 959;

A.Dhar R.Shankar and S.Wadia, Phys. Rev. D31 (1985) 3256

[6] D.Diakonov and V.Petrov, Nucl. Phys. B272 (1986) 457

[7] D.Diakonov, V.Petrov and P.Pobylitsa, Nucl. Phys. B306 (1988) 809;

D.Diakonov and V.Petrov, hep-ph/0009006

[8] Yu.A.Simonov, JETP Lett. 71 (2000) 127, hep-ph/0001244

[9] V.I.Shevchenko and Yu.A.Simonov, Phys. Rev. Lett 85 (2000) 1811, hep-ph/0104135

[10] N.O.Agasian and Yu.A.Simonov, Mod. Phys.Lett. A10 (1995) 1755

[11] N.O.Agasian, Phys. Atom. Nucl. 59 (1996) 297

[12] Yu.A.Simonov, Phys. Lett. B412 (1997) 371

[13] B.O.Kerbikov and Yu.A.Simonov, in preparation

[14] Yu.A.Simonov, Phys. At. Nucl. 60 (1997) 2069; hep-ph/9704301

[15] Yu.A.Simonov, Phys. At. Nucl. 63 (2000) 94

[16] Yu.A.Simonov and J.A.Tjon, Phys. Rev. D62 (2000) 014501

[17] A.Di Giacomo, H.G.Dosch, V.I.Shevchenko and Yu.A.Simonov, hep-ph/0007223, Phys. Rept. (subm. to)

[18] S.V.Ivanov, G.P.Korchensky, Phys. Lett. B154 (1985) 197;

S.V.Ivanov, G.P.Korchensky, A.V.Radyushkin, Sov.Y. Nucl. Phys. 44 (1986) 145

[19] V.I.Shevchenko and Yu.A.Simonov, Phys. Lett. B437 (1998) 146 
[20] I.I.Balitsky, Nucl. Phys. B254 (1985) 166

[21] D.Gromes, Phys. Lett. B115 (1982) 482;

V.Marquard, H.G.Dosch, Phys. Rev. D35 (1987) 2238;

A.Krämer, H.G.Dosch and R.A.Bertlmann, Phys. Lett. B223 (1989) 105

[22] M.Campostrini, A.Di Giacomo and G.Mussardo, Z.Phys. C25 (1984) 173;

A.Di Giacomo and H.Panagopoulos, Phys. Lett. B285 (1992) 133;

A.Di Giacomo, E.Meggiolaro and H.Panagopoulos, preprint IFUP-TH 12/96, heplat/9603017

[23] Yu.A.Simonov, Phys. At. Nucl. 61 (1998) 855; hep-ph/9712248

[24] Yu.A.Simonov, Nucl. Phys. B592 (2001) 350;

M.Eidemueller, H.G.Dosch and M.Jamin, hep-ph/9908318

[25] H.G.Dosch and Yu.A.Simonov, Phys. Lett. B205 (1988) 339

[26] Yu.A.Simonov and J.A.Tjon, Phys. Rev. D62 (2000) 094511; hep-ph/0006237

[27] B.O.Kerbikov and Yu.A.Simonov, preprint ITEP 16-95 (unpublished); hepth/9503146

[28] L.B.Okun, Leptons and quarks, 2nd ed., North-Holland, Amsterdam, 1982.

[29] A.Dhar and S.Wadia, Phys. Rev. Lett. 52 (1984) 959;

[30] D.Ebert and H.Reinhardt, Nucl. Phys. B271 (1986) 188;

[31] Q.Wang et al, hep-ph/9903201

[32] V.D.Mur, V.S.Popov, Yu.A.Simonov and V.P.Yurov, JETP, 78 (1994) 1

[33] S.Weinberg, Phys. Rev. Lett. 67 (1991) 3473;

[34] J.L.Goity and W.Roberts, Phys. Rev. D60 (1999) 034001, hep-ph/9809312 